\title{
MRI-based Microrobotic system for the Propulsion and Navigation of Ferromagnetic Microcapsules
}

\author{
Karim Belharet, David Folio, Antoine Ferreira \\ Institut PRISME, ENSI Bourges, 88 bld Lahitolle, 18020, Bourges, France
}

\begin{abstract}
This paper presents real-time MRI-based control of a ferromagnetic microcapsule for endovascular navigation. The concept was studied for future development of microdevices designed to perform minimally invasive interventions in remote sites accessible through the human cardiovascular system. A system software architecture is presented illustrating the different software modules to allow 3-D navigation of a microdevice in blood vessels, namely: (i) vessel path planner, (ii) magnetic gradient steering, (iii) tracking and (iv) closed-loop navigation control. First, the position recognition of the microrobot into the blood vessel is extracted using Frangi vesselness filtering from the pre-operation images (3-D MRI imaging). Then, a set of minimal trajectory is predefined, using path-planning algorithms, to guide the microrobot from the injection point to the tumor area through the anarchic vessel network. Based on the pre-computed path, a Generalized Predictive Controller (GPC) is proposed for robust time-multiplexed navigation along a two-dimensional (2D) path in presence of pulsative flow.
\end{abstract}

Key words: Blood vessels, magnetic resonance imaging, minimally invasive interventions, real-time control

Email address: antoine.ferreira@ensi-bourges. fr (Antoine Ferreira). 


\section{Introduction}

Microrobots designed to perform targeted therapy by navigating in the cardiovascular system are a prolific research area for minimally invasive surgeries [1][2] and treatments efficiency through early diagnosis of diseases [3]. Cardiovascular problems are generally correlated with the obesity, human sedentary lifestyle, or hereditary characteristics. When microrobots are propelled in the body fluids, especially in the blood circulatory system, a very large number of remote locations in the human body become accessible. However, since the diameters of the blood vessels in the human body may vary from approximately $25 \mathrm{~mm}$ (aorta) down to $0.010 \mathrm{~mm}$ (capillaries), it is obvious that propelling such wireless microdevices in the human cardiovascular system with existing technologies represents a great technical challenge [4]. Because the method of propulsion should allow such a microrobot to navigate through the cardiovascular system, the use of the normal blood flow itself must be considered only as a complementary means of propulsion when the travel path is in the direction of the blood flow. These untethered microrobots have been mainly developed according to three different designs: magnetic bead pulling [2], biomimetic flagellated robot [5] and magnetotactic bacteria [6]. Furthermore, navigation requires observation of the scene in order either to plan the trajectory by off-line mapping, or to correct on-line the microrobot's pose error between the planned and the observed trajectory. Recently, magnetic resonance imaging (MRI)-based medical microrobotic platforms are investigated to reach locations deep in the human body while enhancing targeting efficacy using real-time navigational and trajectory control [7]. For the position recognition of the microrobot in the blood vessels, from the pre-operation images, 3-D path planning and route optimization solutions have been proposed. The authors in [8] proposed an 
endovascular path-planning method based on 3-D potential fields and enhanced breath-first search algorithms based on MR-imaging. In [9], Intra-Vascular UltraSound (IVUS) medical imaging technique coupled to pre-operational images of CT (Computerized Tomography) renders possible 3-D navigation in blood vessels. Based on these path-planning techniques, only explorative 2-D control strategies have been adopted so far using simple proportional-integral-derivative (PID) controller [10]. However, stability and robustness are not ensured against important perturbations. First, pulsatile flow whose variations in waveform, amplitude, and frequency exists from one vessel to another. Second, variation of time-multiplexed sequence parameters (duty cycle of the propulsion gradients, and repetition time of the tracking sequence) produce important trajectory errors during real-time navigation. Finally, random imaging signal noise degrades the localization of the microrobot during tracking.

The main objective of this paper is to propose an automated technique based on image processing and control algorithms for path finding, reconstruction and navigation control of a ferromagnetic microrobot using an MRI system. The MRI-based control of a ferromagnetic microcapsule presented here is dedicated to macroscale navigation, which focuses in conveying the device in vessels such as arteries and arterioles. As illustration of the concept, we consider a possible way for the microrobot get into the body through the femoral artery in the leg, which is the normal access point to the circulatory system. One possible application is to locate atherosclerotic lesions in stenosed blood vessels, particularly in vasculary circulation, and treat them either chemically or pharmacologically by targeted drug delivery. Based on slice images provided by an MRI system, relevant information related to detection of blood vessels is extracted using robust Frangi vesselness filtering from the pre-operation images. Then, a set of minimal trajectory is prede- 
fined, using path-planning algorithms, to guide the ferromagnetic microrobot from the injection point to the tumor area through the anarchic vessel network. Based on the precomputed path, a Generalized Predictive Controller (GPC) is proposed for robust time-multiplexed navigation along a two-dimensional (2D) path in presence of pulsative flow. The simulation results suggest the validation of the proposed image processing and control algorithms. A series of disturbances introduced in the presence and absence of closed-loop control affirms the robustness and effectiveness of this predictive control system.

\section{Methods}

\subsection{MRI-based Microrobotic System Overview}

The Magnetic Resonant Imaging (MRI)-based microrobotic system is used here for propulsion and navigation of the micro-device. The propulsion of the ferromagnetic microcapsule in the cardiovascular system is realized through the induction of force from magnetic gradients provided by the MRI. This MRI system will guide the microcapsule in vivo through vascular networks to a targeted area. MRI-guided microrobotic systems aim at diagnosing and treating diseases in cells, organs or vessels. Their proper function relies on providing engineering and scientific solutions in three technical challenges:

- Enhanced diagnostics: MRI is an advanced imaging system that provides 3D visualization, offering the radiologists a detailed three-dimensional view of the tissue or organ of interest. Microrobotic systems are detectable by MRI without creating artifacts.

- In-vivo propulsion and navigation: The MRI system is employed for propulsion 
Graphical User Interface Module

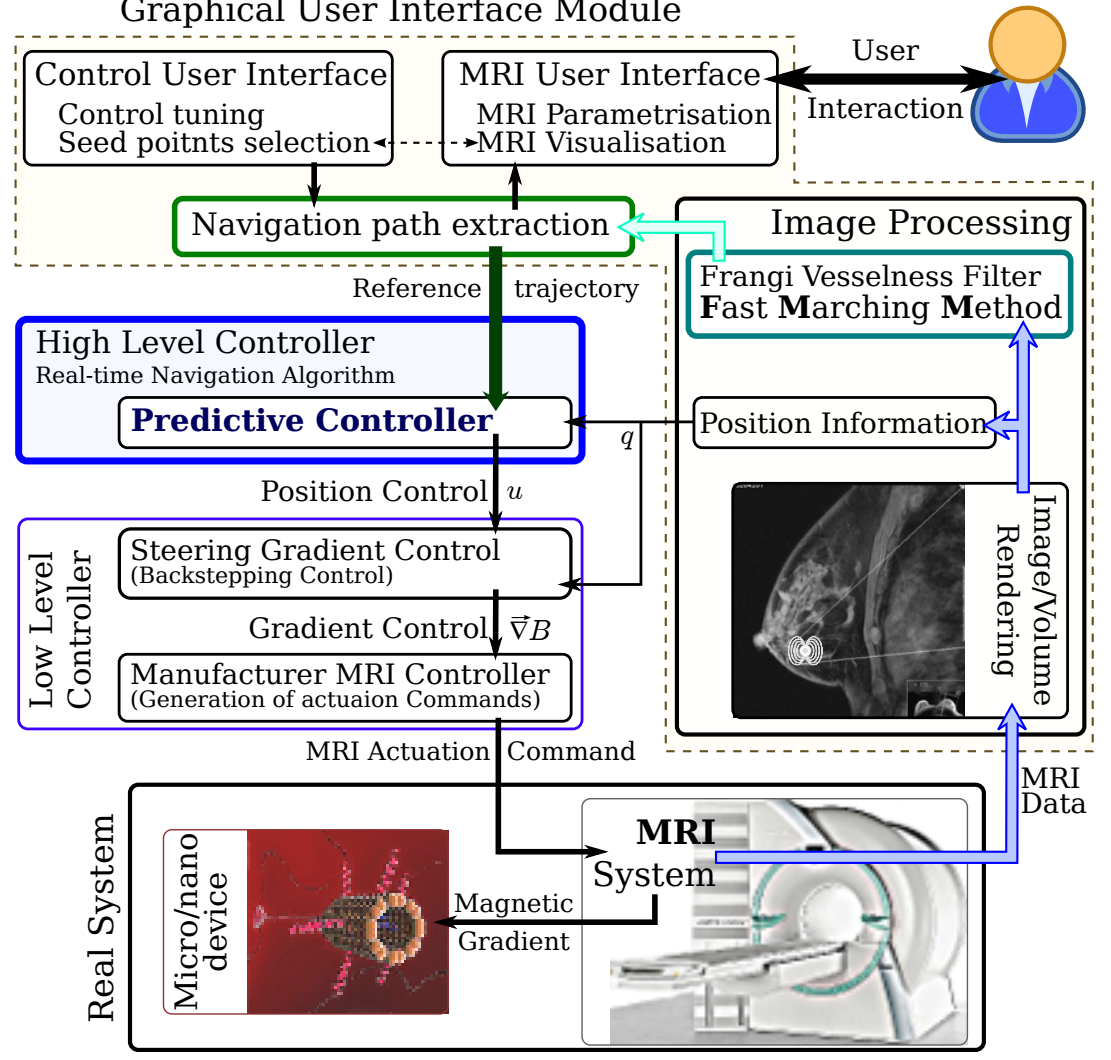

Figure 1. Overall MRI-based microbotic system for steering and navigation of ferromagnetic microcapsules.

and navigation of the microcapsules. The propulsion of a micro or nano-capsule with magnetic properties in the cardiovasular system is realized through the induction of forces and torques by magnetic gradients and magnetic fields respectively, generated by the MRI coils. The microcapsules are guided in-vivo to the targeted organs or are accumulated to the tumors' capillary networks. Also, the MRI forces retain the microcapsules at the target site.

- Drug delivery and release: The magnetic microcapsules are loaded with drug molecules. Moreover, their surface is chemically processed (coated with polymers and bioconjugated with specific antibodies) so that on one hand they are not detected by the immune system and on the other hand they have increased chances to bind to the receptors of malignant cells. When the microcapsules are 
inside the cell or at its vicinity, the release of the drug is triggered.

The overview of the software system architecture is given in Fig. 1. (i) The graphical user interface module, which comprises input command prompt, 3D-visualization, and process supervision tools. (ii) The control module, which comprises (a) the high-level controller responsible for the microcapsule navigation tasks and for the generation of the magnetic field gradients and (b) the low-level controller (manufacturer MRI controller) responsible for implementing the actuation commands for the generation of the desired field gradients and for the image acquisition tasks. Then, (iii) the controlled hardware, which comprises (a) the MRI hardware and software systems and (b) the microcapsules that have been injected within the vasculature and are navigated by the field gradients. Finally, (iv) the image processing module, which comprises the (a) MRI image reconstruction and (b) the imageprocessing software that estimates the position and accumulation of the microcapsules within the vasculature, the tissues, and the organs of the human.

\subsection{Finding Endovascular Navigation Path}

In our context, the problem of finding the navigation path within the MRI data can be formulated as finding the correct way through the data which follows the vessel of interest between its start and end point. Finding a navigation path within the vessel network is then an essential, primary, and important step which must be addressed prior to the control procedure. The problem of vessels extraction has received considerable attention in the computer vision and medical imaging communities [11]. Hence, several class of methods have been proposed to find a path from a set of medical imaging, such as using tracking methods [12] [7] [13], path extraction methods [14][15][16], and so on. Most works based on in vivo MR-tracking 
methods usually need many user-defined way points as the input of a controller module for the navigation computation. A major drawback in general remains when the user must define many points (e.g. way or fiducial points) manually. Hence, for a complex structure (e.g. colon, small vessels...) the required interactivity can be very tedious. As consequence, if the path is not correctly build, it can cross an anatomical wall during the in vivo navigation. In opposition, minimal path extraction approaches depend only on the manual definition of the start and end points of the desired vascular path. Therefore, we propose in this work to use path extraction technique to find the desired path to be followed in the vessel network.

The path extraction is useful for a range of application domains including medical image analysis, robot navigation, and artificial intelligence. The problem of finding the trajectory in the imaging data can be defined as finding the correct path through the data that follows the vessel of interest between its start and end point. The minimal path technique has many advantages. It needs a very simple initialisation and leads to global minimum of a snake-like energy, thus avoiding local minima. Moreover it is fast and accurate. This minimal path problem has been studied for ages by mathematicians, and has been solved numerically using graph theory or dynamic programming. Several authors have addressed the minimal path extraction approaches. For instance, Cohen and Kimmel [17] solved the minimal path problem in 2D with a front propagation equation between the two fixed end points, using the Eikonal equation (that physically models wave-light propagation), with a given initial front. Wink et al.[15] explored different methods to determine the minimum cost path through a pre-defined cost image, for extraction of vessel centrelines from medical image data. Among them are Dijikstra's algorithm [18], the $A^{\star}$ algorithm [19], which makes use of additional heuristics to steer the search process, and wave front propagation analysis [16]. Early, Sethian [20] explore the use 
of Fast Marching Method (FMM) to extract minimal paths. This method relies on the fact that the gradient of the FMM arrival function has only one local minimum, with is guaranteed to be global minimum [14]. Therefore the minimal path can be extracted by back-propagating from given seed (corresponding to the end point of the desired path) to the starting point implicitly embedded in the arrival function. In this work the FMM is adopted to design a set of trajectory to guide the microdevice from the injection point to the tumor area through the vessel network. Our aim is to focus on the automation of the path construction, reducing the need of interaction and improving performance, in a robust way. Finally, as the proposed control strategy, presented in section 3.2, is designed for $2 \mathrm{D}$ navigation problem, we have limited our path extraction procedure to $2 \mathrm{D}$ application.

\subsection{Path Extraction Results}

The FMM algorithm, introduced by Sethian [20] is applied here to extract a targeted navigation path within the vessel network. The FMM is very closely related to Dijkstra's method [18], which is a very well-known method from the 1950's for computing the shortest path on a network. Hence, from the set of MRI data we have first to compute a speed map (ie. a weighting image map), which must enhance the relevant intravascular network. Choosing an appropriate and efficient image cost function is the most difficult part of the entire process. The definition of the optimal speed function is case dependent and should be set by the user, along with the start and end points of the path. Hence, an optimal path could be found only if the optimal cost function is provided. In this work, we focus mainly on finding a minimal path allowing reaching the targeted zone.

We describe in the sequel presented in Fig. 2, the process used to extract naviga- 


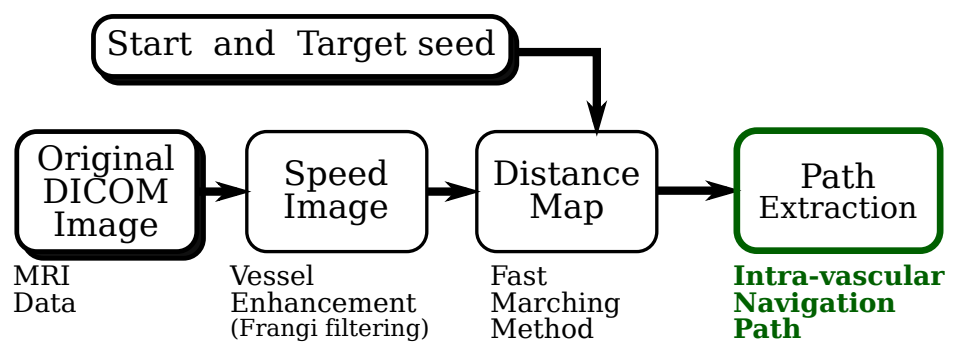

Figure 2. Image processing pipeline.

tion path. First, we need a relevant cost function which allow to enhance vessel in the image. To this aim we use some a priori knowledge about vessel shape and intensity in MRI data (see Fig. 3). Vessels are expected to appear as bright tubular structures in a darker environment. One way to account for the varying size of vessels is by multiscale analysis. It allows us to detect structures of different sizes according to the scale at which they give maximal response. In this context, a typical speed image is produced by using a Frangi vesselness filter [21] which uses the eigenvectors of the Hessian matrix at each voxel of the image to compute the likeliness of an image region to vessels. This mapping is selected in such a way that vessels regions will have higher speed (high level in speed image, see Fig. 3). Once the speed map is generated, the user has to select a start and end points (ie. seed points) in the viewer of the input original image. The FFM will then propagate a front from the start seed and traveling to the targeted area, thanks to the speed map. This step allow to build an image of distance between the start seed and all other pixels. The corresponding shortest path is then traced thanks to the distance map.

We have applied the described approach on some representative MRI data which validate our image processing pipeline. The application is to locate atherosclerotic lesions in stenosed blood vessels, particularly in vascular circulation. We consider that the microrobot get into the body through the femoral artery in the leg, and treat them pharmacologically, by tageted drug delivery (see Fig. 3). Let us also notice 


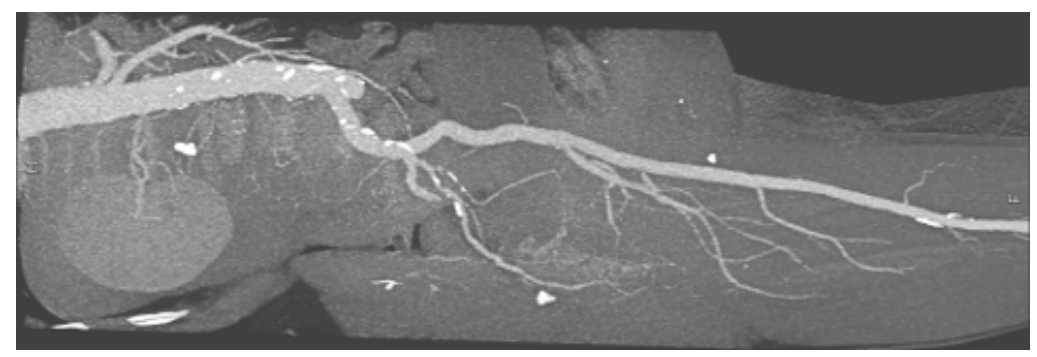

(a)

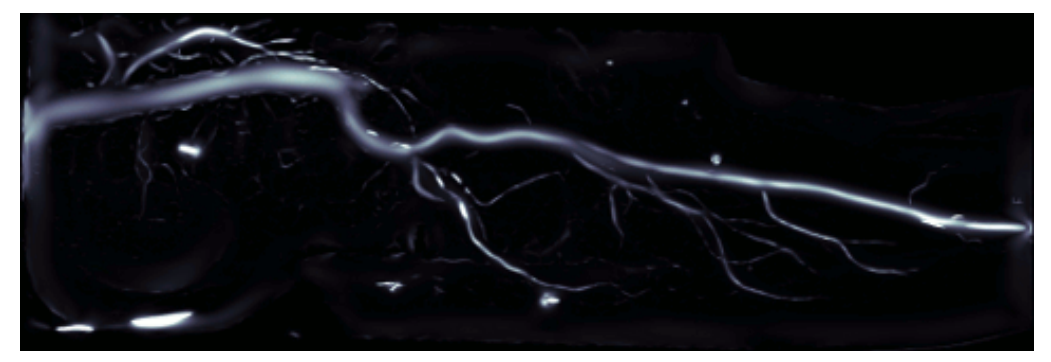

(b)

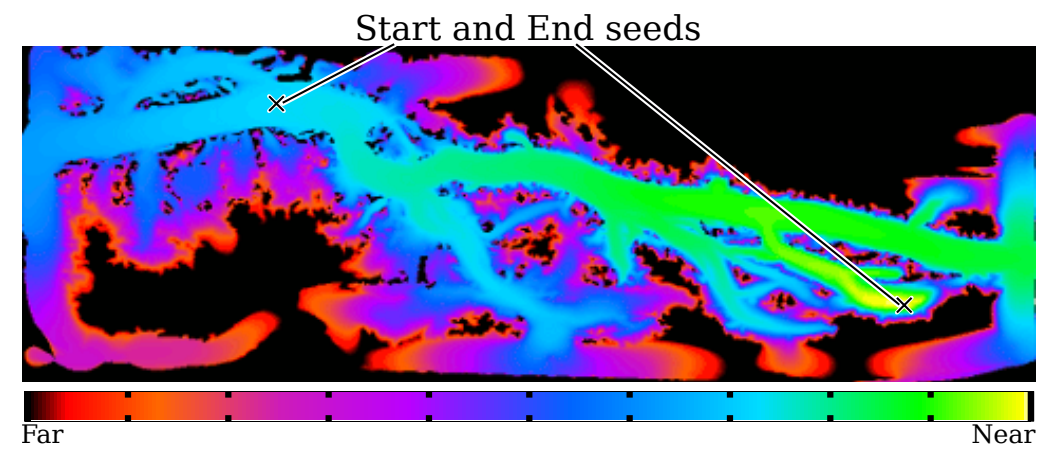

(c)

Start and End seeds

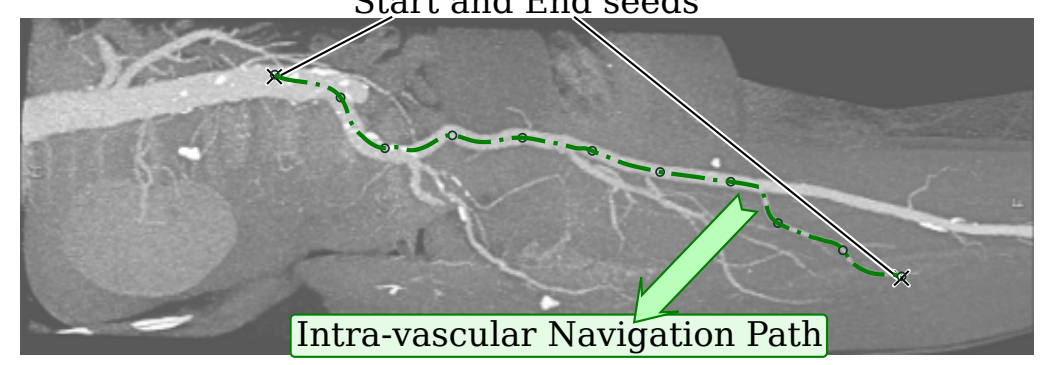

(d)

Figure 3. 2-D Navigation path extraction processing of atherosclerotic lesions in the upper right leg: a) Original MRI data; b) Computed cost function using Frangi vesselness filter (light pixel have higher weight); c) FFM propagation to build the distance to targeted seed map; d) Computed navigation path between the start and end seeds. 


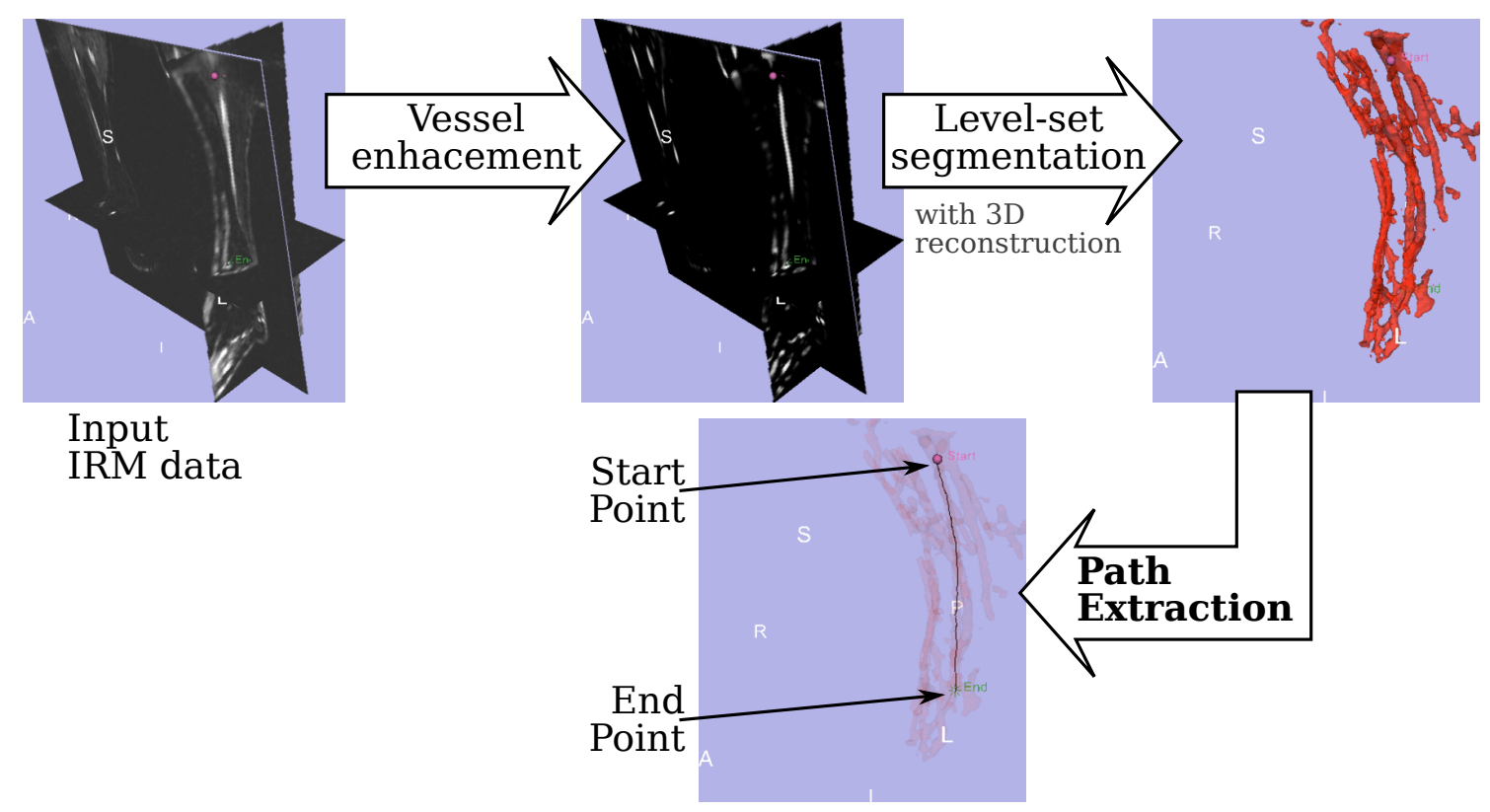

Figure 4. 3-D Navigation path extraction processing of atherosclerotic lesions in the upper right. leg.

that the proposed image processing pipeline (see Fig. 2) could be applied to 3D MRI data, and then provide 3D navigation path. A MRI system generates slice images sequentially of a specific zone. Due to the fact that the body is in motion, there may be discontinuities between two images generated by these motion artifacts. In order to setup a 3D volume of the images, the images are parsed and relevant information is extracted. Once the images are reconstructed, the 3D data structures and filters may be applied to the whole volume. Applying the proposed image processing pipeline, 3D navigation path extraction is rendered possible. The Fig. 4 shows the $3 \mathrm{D}$ navigation path extraction processing steps. A close view of all possible paths along the blood vessel network is presented in Fig. 5. 


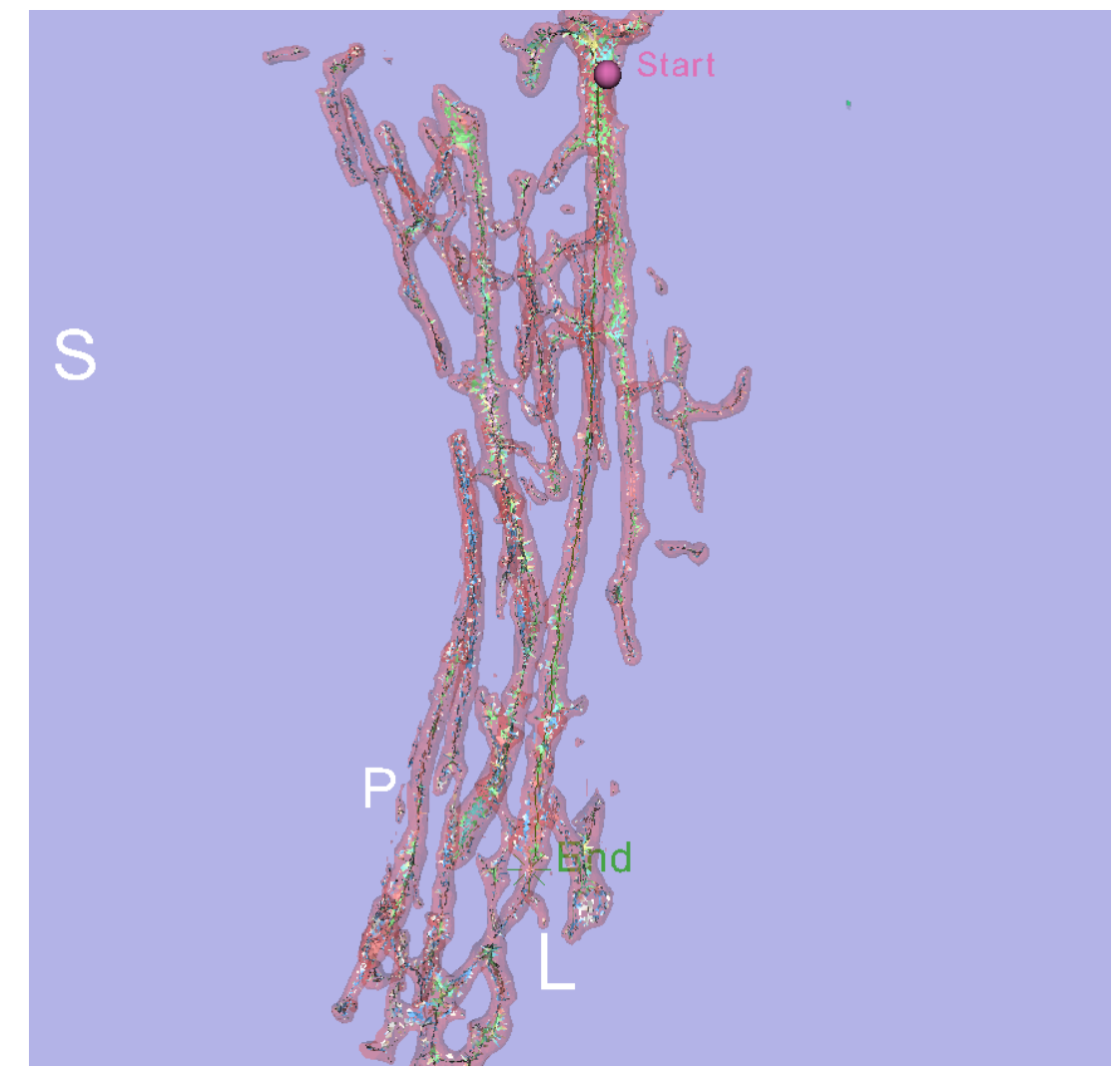

Figure 5. Path extraction in blood vessel network of upper right leg.

\section{CONTROL DESIGN}

\subsection{Problem formulation}

Endovasular navigation requires observation of the scene in order either to plan the trajectory by off-line mapping, or to correct online the microrobot's pose error between the planned and the observed trajectory. To insure a smooth conveyance of the microcapsule to destination, collisions and the risk to be trapped by the endothelium, optimal navigation performance will be affected by external perturbations and MRI technological constraints:

- Nonnegligible pulsatile flow, whose variations in waveform, amplitude, and frequency exist from one vessel to another (such as arteries and arterioles). 
- Magnetic gradients are used both for observation and control purposes in a timemultiplexed sequence. It requires different trade-offs in terms of refresh rate, duty cycle of the propulsion gradients, and repetition time of the tracking sequence.

- MRI overheating avoidance leading to limitations on the MRI duty cycle, tends to increase the disproportional scaling between magnetic forces used for control purpose and perturbation forces (drag forces and net buoyancy forces).

- Limitations on the magnetic gradient amplitude in available MRI devices.

- Proper delay in the image processing algorithms that renders the navigation control unstable.

\subsection{Real-Time Sequence Design}

The overall concept of the in-vivo MRI-tracking system is based on the fact that both tracking and propulsion is possible with the gradient coils of the MRI system. Software based upgrading of a clinical MRI system is the least expensive approach to convert a platform that is used for imaging to an effective interventional platform. At any instant only of the functions could be applied (i.e. either tracking or propulsion), but both will be executed over the same MRI interface.

The MRI interface has therefore to be shared and a time-division-multiple-access scheme for it has to be developed. Fig. 6 shows an overview of the real-time sequence with time-multiplexed positioning and propulsion phases introduced by Martel et al. [7]. The main aspect relevant to the controller's performance is (i) the duty cycle $T_{\text {Prop }} / T_{S}$ that stands for the ratio between the propulsion time and the time between two successive position requests, and (ii) the synchronization event delay 


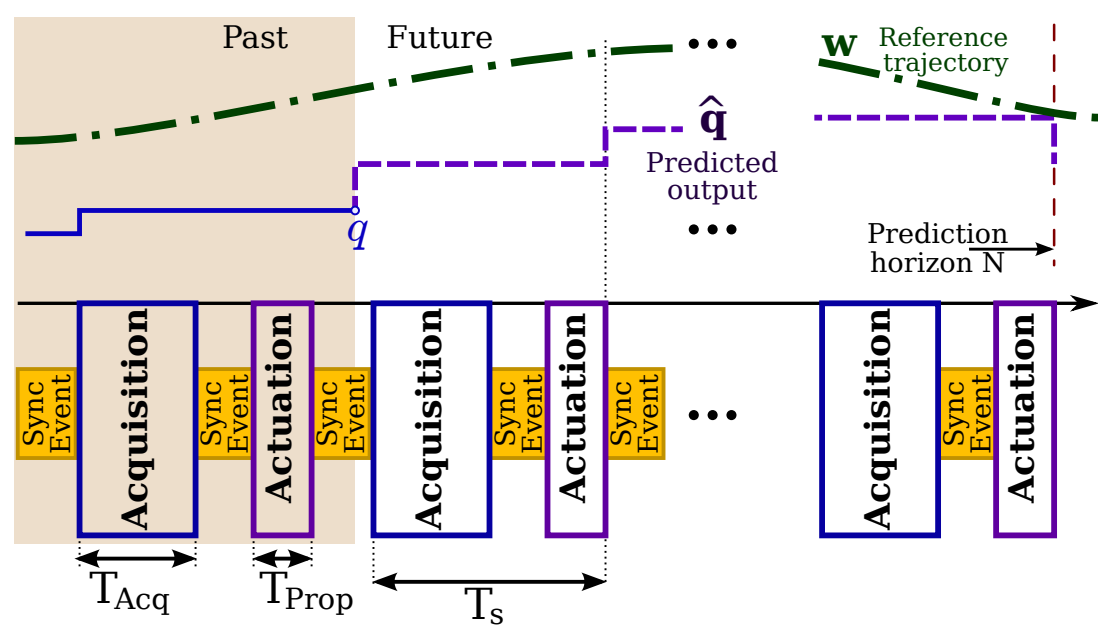

Figure 6. Timeline of acquisition and control prediction.

$T_{\text {sync }}$ that stands for the minimum time allowed for image processing and real-time control feedback. First, the duty cycle should be adapted to apply sufficient magnetic propulsion gradients during a predefined propulsion time $T_{\text {Prop }}$ to prevent the microrobot from drifting away from the trajectory. Second, a large time delay $T_{\text {sync }}$ produces oscillations as the microrobot approaches the reference trajectory leading to position instabilities. Such limitations have been pointed out by Mathieu et al. [10] when implementing simple proportional-integral-derivative (PID) controller. We proposed a navigation Generalized Predictive Controller (GPC) including microrobot's motion and dynamics with estimation of the pulsative blood flow and time-multiplexed positioning. A predictive trajectory-tracking control consider a prediction window varying typically from 10 to 50 sampling intervals. The propulsion phase starts during $T_{\text {Prop }}$ seconds at the same initial condition as the prediction phase, recording the performance of the system according to a prediction horizon. After this phase the system ends after a imaging-propulsion sequence at a final position $q$ which is set as the new initial condition of the next prediction output $\hat{q}$. The proposed navigation based predictive controller offers stability by design and allows the designer to trade-off performance for (computation) speed, stability margins according to the MRI application and technological requirements outlined in 
section 3.1 .

\subsection{Model description}

The linear model that was used in this work, derived from the nonlinear model developed in a previous study [22]. In [22], we used this model to combine the backstepping controller and high gain observer in order to control the trajectory of microrobot inside a vessel using the MRI gradients.

The different forces acting on the microrobot are (see figure 7): drag force $\vec{F}_{d}$,

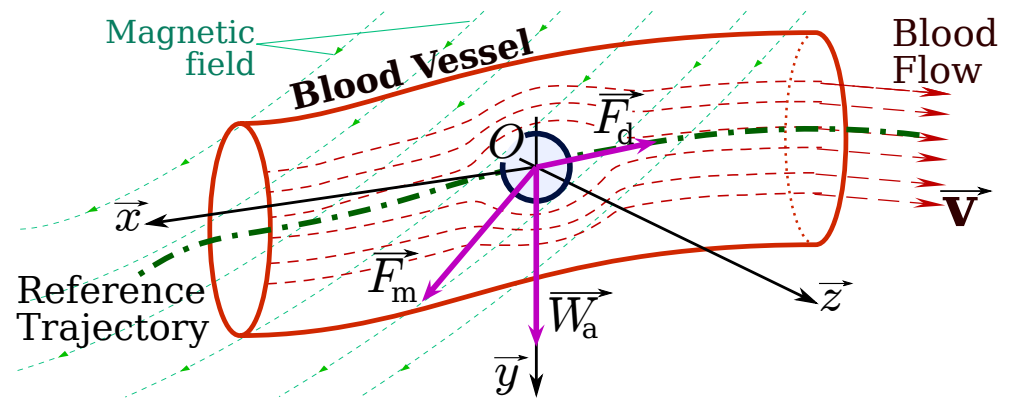

Figure 7. Forces applied on microrobot.

apparent weight $\vec{W}_{a}$ and magnetic force $\vec{F}_{m}$. The application of Newton's third law and the projection on the $\vec{x}$-axis and $\vec{y}$-axis leads to:

$$
\left\{\begin{array}{l}
m \ddot{x}=\vec{F}_{d x}+\vec{F}_{m x} \\
m \ddot{y}=\vec{F}_{d y}+\vec{F}_{m y}+\vec{W}_{a}
\end{array}\right.
$$

where $m$ is the mass of the microrobot.

Let $\overrightarrow{\mathbf{v}}=\left(v_{f_{x}}, v_{f_{x}}\right)$ denotes the blood flow velocity, and $(x, y)$ the robot location in the blood vessel wrt. to a given frame $\mathscr{F}(O, \vec{x}, \vec{y})$. Taking the drag coefficient 
$C_{d}=\frac{24}{R e}$, the linear model can be written as follow:

$$
\left\{\begin{array}{l}
\ddot{x}=\alpha_{1}\left(\dot{x}-v_{f_{x}}\right)+\alpha_{2} u_{x} \\
\ddot{y}=\beta_{1}\left(\dot{y}-v_{f_{y}}\right)+\beta_{2} u_{y}
\end{array}\right.
$$

with the following parameters $\alpha_{i}$ and $\beta_{i}$, and the magnetic gradients considered as control inputs $u_{x}$ and $u_{y}$, that is:

$$
\begin{cases}\alpha_{1}=-4.5 \frac{\eta \cos \theta}{r^{2} \rho}, & u_{x}=\left\|\vec{\nabla} B_{x}\right\| \\ \beta_{1}=-4.5 \frac{\eta \sin \theta}{r^{2} \rho}, & u_{y}=\left\|\vec{\nabla} B_{y}\right\| \\ \alpha_{1}= & \beta_{2}=\frac{M}{\rho}\end{cases}
$$

where $\rho$ is the density of the fluid; $\eta$ is the fluid viscosity; $r$ is the spherical radius; and $\mathbf{B}=\left(B_{x}, B_{y}\right)^{T}$ is the magnetic field generate by the MRI system.

Finally, the state space representation is deduce from (2):

$$
(S)\left\{\begin{array}{l}
\dot{x}=v_{x} \\
\dot{v}_{x}=\alpha_{1} v_{x}-\alpha_{1} v_{f_{x}}+\alpha_{2} u_{x}
\end{array}\right\}\left(S_{x}\right)
$$

where $\left(v_{x}, v_{y}\right)^{T}$ denote the robot velocity along $x$-axis and $y$-axis. Assuming that microrobot location $(x, y)$ can be measured thanks to the MRI system, we denote by $q=(x, y)^{T}$ the process measure. We can notice that system $(S)$ can be divided into two subsystems $\left(S_{x}\right)$ and $\left(S_{y}\right)$, which allow us to define two independents GPC schemes to track the reference trajectory in 2D MRI data. 


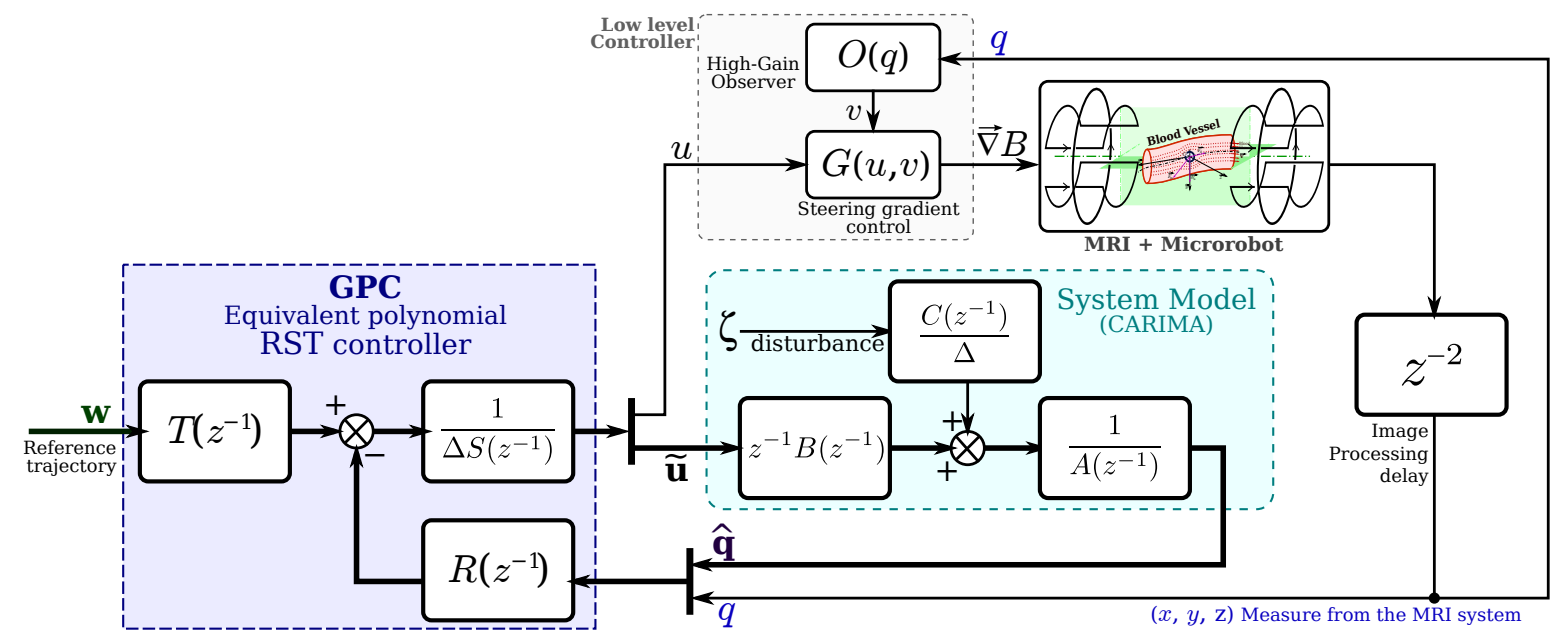

Figure 8. Navigation based-Generalized Predictive Control (GPC) strategy..

\subsection{Generalized Predictive Control (GPC)}

Generalized Predictive Control (GPC) belongs to the class of Model Predictive Control (MPC) techniques and was first introduced by Clarck et al.[23,24]. GPC approach is a popular control predictive method, experienced on number various applications [25] [26], especially in industrial process [27]. It combines the prediction of future behavior of the system with feedback control (see figure 8). A process model is explicitly used to predict the future behavior of the system over the given time horizon. From this prediction, a control is computed by minimizing a quadratic cost function.

\subsubsection{MIMO GPC Scheme}

The system is modeled using the model Controlled Auto-Regressive Integrated Moving-Average (CARIMA) with integrator form, that is [23]:

$$
A\left(z^{-1}\right) q(t)=B\left(z^{-1}\right) u(t-1)+C\left(z^{-1}\right) \frac{\zeta(t)}{\Delta\left(z^{-1}\right)},
$$


where $\Delta\left(z^{-1}\right)=1-z^{-1}$ define the difference operator; $\zeta(t)$ is a zero mean white noise; and $A\left(z^{-1}\right), B\left(z^{-1}\right)$ and $C\left(z^{-1}\right)$ are polynomial matrix in the backward shift operator $z^{-1}$, for order $n_{A}, n_{B}, n_{C}$ we get:

$$
\begin{aligned}
& A\left(z^{-1}\right)=1+a_{1} z^{-1}+\ldots+a_{n} z^{-n_{A}} \\
& B\left(z^{-1}\right)=1+b_{1} z^{-1}+\ldots+b_{n} z^{-n_{B}} \\
& C\left(z^{-1}\right)=1+c_{1} z^{-1}+\ldots+c_{n} z^{-n_{C}}
\end{aligned}
$$

The GPC control strategy is obtained by minimizing a weighted sum of square predicted future errors and square control signal increments:

$$
J_{\left\{N_{1}, N_{2}, N_{u}, \lambda\right\}}=\sum_{j=N_{1}}^{N_{2}}(\widehat{y}(t+j)-w(t+j))^{2}+\lambda \sum_{j=1}^{N_{u}} \Delta u^{2}(t+j-1)
$$

where $\widehat{y}_{t+j}$ is the optimum predicted output of the system at time $t+j ; w_{t+j}$ is the future reference; $N_{1}$ and $N_{2}$ are the minimum and the maximum of the prediction horizon. $N_{u}$ is the control horizon; and $\lambda>0$ is the control increment weighting. $N_{1}, N_{2}, N_{u}$ and $\lambda$ are the design parameters of the GPC.

The optimal predictor built from the measured output data up to time $t$ and any given $u(t+j)$ for $j>1$ is:

$$
\widehat{\mathbf{q}}=\mathbf{G} \widetilde{\mathbf{u}}+\mathbf{f}\left(z^{-1}\right) q(t)+\mathbf{h}\left(z^{-1}\right) \Delta u(t-1)
$$

where $\mathbf{f}\left(z^{-1}\right)$ and $\mathbf{h}\left(z^{-1}\right)$ are the polynomial calculated by solving the Diophantine equations:

$$
\left\{\begin{array}{l}
A\left(z^{-1}\right) J_{j}\left(z^{-1}\right) \Delta\left(z^{-1}\right)+z^{-1} F_{j}\left(z^{-1}\right)=1 \\
B\left(z^{-1}\right) J_{j}\left(z^{-1}\right)=G_{j}\left(z^{-1}\right)+z^{-1} H_{j}\left(z^{-1}\right)
\end{array}\right.
$$




$$
\begin{aligned}
& \mathbf{f}=\left[F_{N_{1}}\left(z^{-1}\right) \ldots F_{N_{2}}\left(z^{-1}\right)\right]^{T} \\
& \mathbf{h}=\left[H_{N_{1}}\left(z^{-1}\right) \ldots H_{N_{2}}\left(z^{-1}\right)\right]^{T} \\
& \widetilde{\mathbf{u}}=\left[\Delta u(t) \ldots \Delta u\left(t+N_{u}-1\right)\right]^{T} \\
& \widehat{\mathbf{q}}=\left[\begin{array}{lll}
\widehat{q}\left(t+N_{1}\right) & \ldots \widehat{q}\left(t+N_{2}\right)
\end{array}\right]^{T} \\
& \mathbf{G}=\left[\begin{array}{cccc}
g_{N_{1}}^{N_{1}} & g_{N_{1}-1}^{N_{1}} & \ldots & \\
& & & \\
g_{N_{1}+1}^{N_{1}+1} & g_{N_{1}}^{N_{1}+1} & \ldots & \\
\vdots & \vdots & & \\
& & & \\
g_{N_{2}}^{N_{2}} & g_{N_{2}-1}^{N_{2}} & \ldots & g_{N_{2}-N_{u}+1}^{N_{2}}
\end{array}\right]
\end{aligned}
$$

where $G_{j}$ represents the future; $F_{j}$ and $H_{j}$ define respectively the present and the past; and $J_{j}$ is related to the disturbance.

Hence, the optimal control is defined by:

$$
\widetilde{\mathbf{u}}=\mathbf{M}\left[\mathbf{w}-\mathbf{f}\left(z^{-1}\right) q(t)-\mathbf{h}\left(z^{-1}\right) \Delta u(t-1)\right]
$$

with $\mathbf{w}=\left[w\left(t+N_{1}\right) \ldots w\left(t+N_{2}\right)\right] ; \mathbf{M}=\mathbf{Q G}^{T}$ with dimension $N_{u} \times\left(N_{2}-N_{1}+1\right)$; and $\mathbf{Q}=\left[\mathbf{G}^{T} \mathbf{G}+\lambda \mathbb{I}_{N_{u}}\right]^{-1}$ with dimension $N_{u} \times N_{u}$. Only the first element of $\widetilde{\mathbf{u}}$ is applied to the system, that is:

$$
\Delta u^{\mathrm{Opt}}(t)=\mathbf{m}_{1}^{T}\left[\mathbf{w}-\mathbf{f}\left(z^{-1}\right) q(t)-\mathbf{h}\left(z^{-1}\right) \Delta u(t-1)\right]
$$

where $\mathbf{m}_{1}^{T}$ is the first row of $\mathbf{M}$.

Classically a RST polynomial structure is introduced at the end to determine a relation between the output $q(t)$, the control signal $u(t)$ and the setpoint $w(t)$, according to the scheme of Fig.8. The advantage of RST polynomial structure is that 
these modules can be computed off-line, providing a very short real-time loop and on the other hand offers the possibility to analyze the stability of the controlled open loop in the frequency domain. In fact, this off-line operation is a very helpful strategy to determine the stable set of tuning parameters just before applying the control law on the real system. Hence the above control law (11) can be rewritten in a RST polynomial form:

$$
S\left(z^{-1}\right) \Delta\left(z^{-1}\right) u(t)=T\left(z^{-1}\right) w(t)-R\left(z^{-1}\right) q(t)
$$

\subsubsection{GPC implementation}

In order to design the GPC controller the transfer functions of the two subsystems $\left(S_{x}\right)$ and $\left(S_{y}\right)$ of Eq.(4) are obtained:

$$
\begin{aligned}
& H_{x}(s)=\frac{243.8}{s^{2}+49.25 s} \\
& H_{y}(s)=\frac{243.8}{s^{2}+79.77 s}
\end{aligned}
$$

The GPC controller under RST polynomial form requires tuning of the set parameters $N_{1}, N_{2}, N_{u}$ and $\lambda$ to ensure good stability. Some guidelines may be found in the literature [28]. For this process, two independent GPC controllers has been designed for a sampling period of $T_{\mathrm{s}}=50 \mathrm{~ms}$, satisfying stability and robustness features [28], with the tuning parameters:

$$
\left\{N_{1}=1 ; N_{2}=4 ; N_{u}=1 ; \lambda_{x}=0.77, \lambda_{y}=0.34\right\}
$$

According to these parameters, the model of the process using (13) and (14) in 
CARIMA (5) form is:

$$
\begin{aligned}
& \left(S_{x}\right)\left\{\begin{array}{l}
A_{x}\left(z^{-1}\right)=\left[1-1.085 z^{-1}+0.085 z^{-2}\right], \\
B_{x}\left(z^{-1}\right)=\left[0.155+0.071 z^{-1}\right]
\end{array}\right. \\
& \left(S_{y}\right)\left\{\begin{array}{l}
A_{y}\left(z^{-1}\right)=\left[1-1.018 z^{-1}+0.018 z^{-2}\right] \\
B_{y}\left(z^{-1}\right)=\left[0.115+0.034 z^{-1}\right]
\end{array}\right.
\end{aligned}
$$

and where $C_{x}\left(z^{-1}\right)$ and $C_{y}\left(z^{-1}\right)$ are set to 1 .

The RST form optimal control is:

$$
\begin{aligned}
& \Delta u_{x}^{\mathrm{Opt}}(t) S_{x}\left(z^{-1} \neq-R_{x}\left(z^{-1}\right) q_{x}(t)+T_{x}\left(z^{-1}\right) w_{x}(t)\right. \\
& \Delta u_{y}^{\mathrm{Opt}}(t) S_{y}\left(z^{-1} \neq-R_{y}\left(z^{-1}\right) q_{y}(t)+T_{y}\left(z^{-1}\right) w_{y}(t)\right.
\end{aligned}
$$

with

$$
\begin{aligned}
& \left\{\begin{array}{l}
R_{x}\left(z^{-1}\right)=2.855-2.222 z^{-1}+0.169 z^{-2} \\
S_{x}\left(z^{-1}\right)=1+0.140 z^{-1} \\
T_{x}\left(z^{-1}\right)=0.123 z+0.273 z^{2}+0.405 z^{3}
\end{array}\right. \\
& \left\{\begin{array}{l}
R_{y}\left(z^{-1}\right)=4.0 .61-2.906 z^{-1}+0.052 z^{-2} \\
S_{y}\left(z^{-1}\right)=1+0.098 z^{-1} \\
T_{y}\left(z^{-1}\right)=0.206 z+0.410 z^{2}+0.592 z^{3}
\end{array}\right.
\end{aligned}
$$




\subsection{Simulation Results}

Simulations are conducted within the scope of actual commonly spread MRI system abilities. At the moment, MRI systems are able to generate magnetic gradients with an intensity of some tens of $m T \cdot m^{-1}$. Let us note that this limitation is additionally affected by the gradient coils duty cycle and by the multiplexing needed both for controlling and observing. In order to make sure that the amplitude of the control inputs remains bounded by physical actuators limits $u_{i, \max }$ and to protect the system, we perform a simple time scaling. Thus, the applied control law

is given by $\frac{u_{i}}{k(t)}$, with $k(t)=\max \left\{1, \frac{u_{i}}{u_{i, \max }}\right\}$. The set of simulations corresponds to microcapsule's radius of $300 \mu \mathrm{m}$ and a time scaling settled at $k(t)=0.55$. Different situations are considered in this section to illustrate and validate the performance and robustness of the proposed MRI-based predictive controller shown on Fig. 8 . As the considered system is decoupled into two subsystems $\left(S_{x}\right)$ and $\left(S_{y}\right)(4)$, we have first to validate the control strategy onto 1D longitudinal path. As illustration, the microcapsule has to follow a sinusoidal reference trajectory (cf. Fig. 9 and 10) for different time horizons $N=N_{2}-N_{1}$ (namely $N=3,10$, and 15).

Figure 9 presents the trajectories followed by the microrobot, and the relative error between the current position $q$ and the reference path $w$. As one can see the system output follows correctly the reference trajectory $w$ for each considered time horizon. The output of the closed loop system is dependent on the setting parameters of the GPC. The previous curves show the impact of the prediction horizon $N$ for the controlled system. Moreover, comparing both plots, the nature of anticipation of the GPC scheme is illustrated by -greater is $N$ more is anticipated the path behavior-. Hence, a great value of $N$ does not necessarily guarantee good performance, and generally increase the complexity of the scheme. To evaluate the efficiency of the 

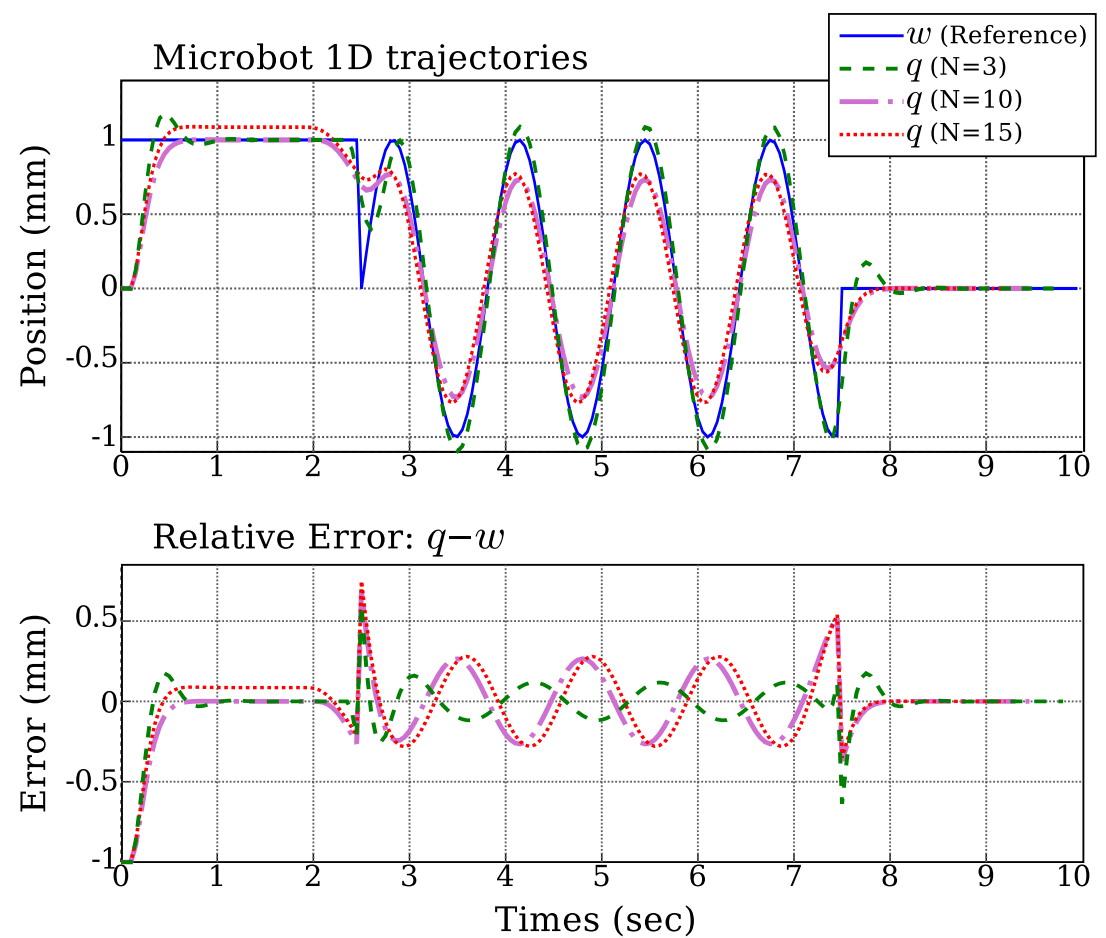

Figure 9. Longitudinal position microrobot control.
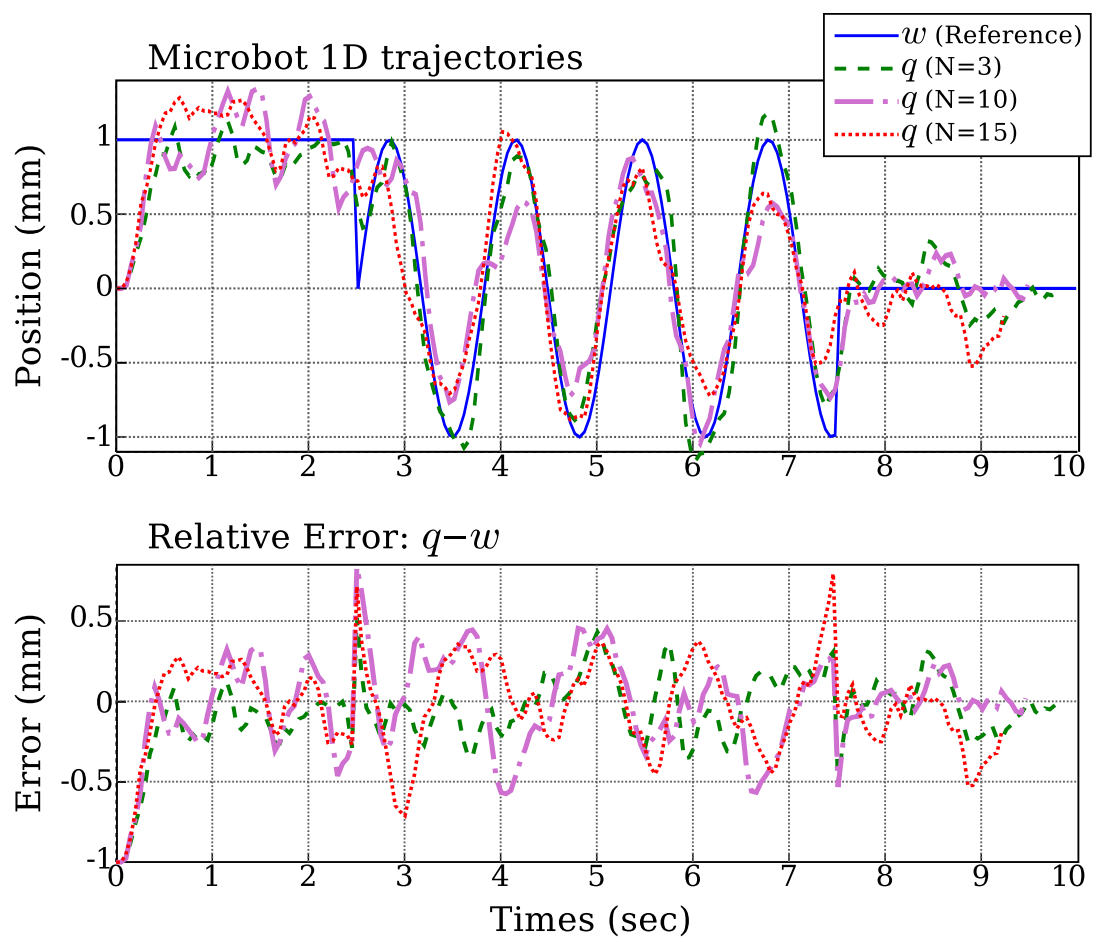

Figure 10. Longitudinal position microrobot control with white noise. 
proposed MRI-based predictive controller, we added a white Gaussian noise on the system output measure $q$. Fig. 10 shows the system response in the presence of this disturbance on the system. Globally, tracking is not too much affected by the noise, since position standard deviation $(s t d)$ and root mean square $(R M S)$ error are quite satisfactory (see Table 1).

Table 1

Error statistics

\begin{tabular}{|l||lll||l|l|}
\hline \multicolumn{1}{|l||}{} & \multicolumn{3}{c||}{ 1D navigation with noise } & \multicolumn{2}{c|}{ 2D navigation } \\
& $N=3$ & $N=10$ & $N=15$ & without noise & with noise \\
\hline \hline std & 0.2723 & 0.2887 & 0.2976 & 1.0438 & 1.2121 \\
\hline RMS & 0.2753 & 0.3068 & 0.3153 & 1.0480 & 1.2306 \\
\hline
\end{tabular}

We validated the proposed control strategy on $2 \mathrm{D}$ endovascular navigation path extracted from MRI-data with the method presented in section III.4. As shown in Fig. 11, the system output $q$ follows perfectly the reference trajectory $w$ without (cf. Fig. 11(a)) and with (cf. Fig. 11(b)) white noise added. In particular, the microrobot is able to converge quickly towards the navigation path, in spite of a large gap between the injection point and the starting of reference path $w$ (about 50 pixels). Fig. 12 describes the error evolution in both cases. Once again the error remains small with low values of std and RMS parameters. Let us notice that these $2 \mathrm{D}$ error statistics given in table 1 take into account the gap between the injection point and the initial reference path. 


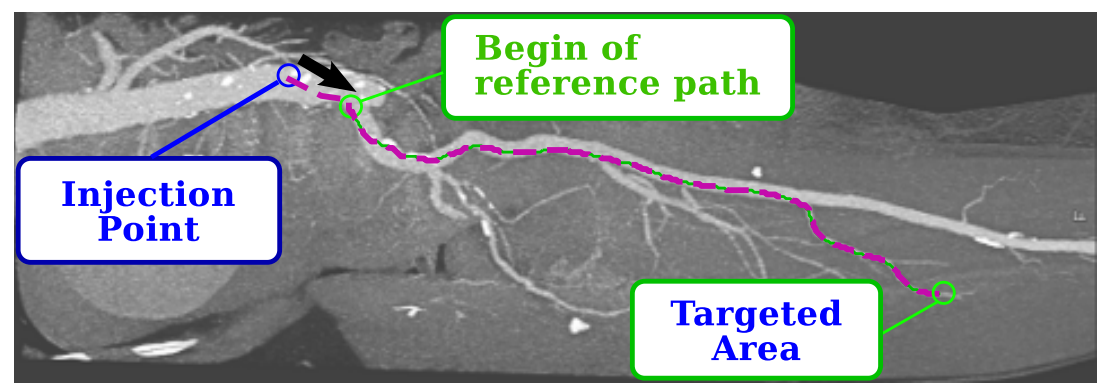

(a) Trajecrory without noise.

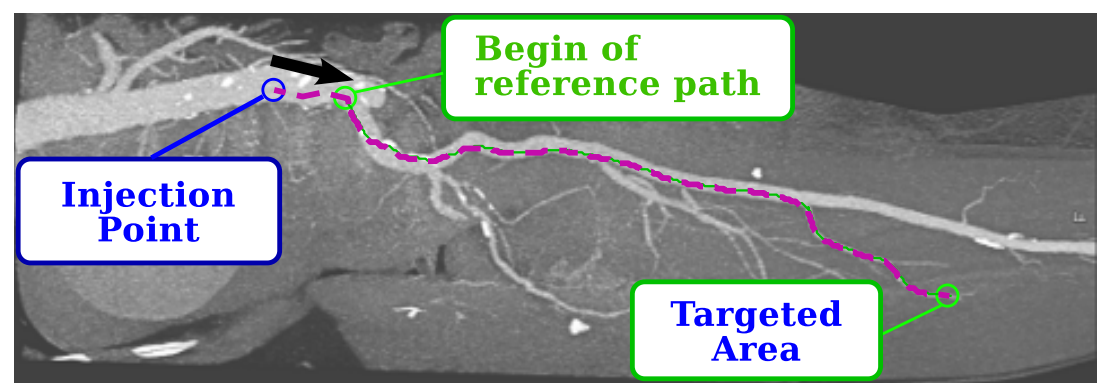

(b) Trajectory with white noise.

Figure 11. 2D MRI-based microrobot endovascular navigation $(N=3)$.

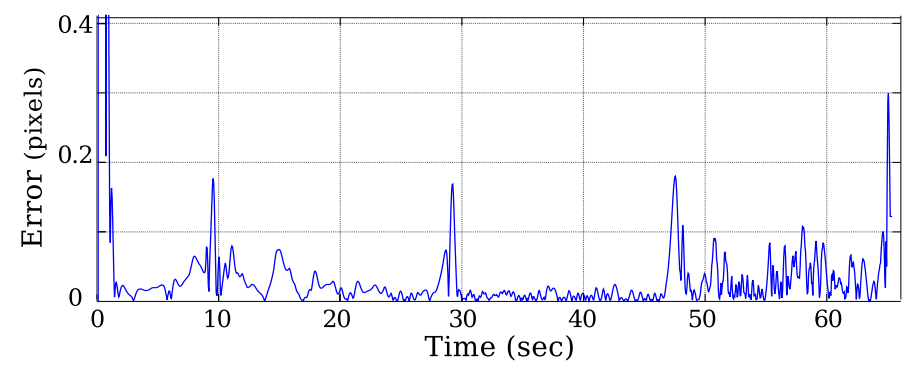

(a) Without noise.

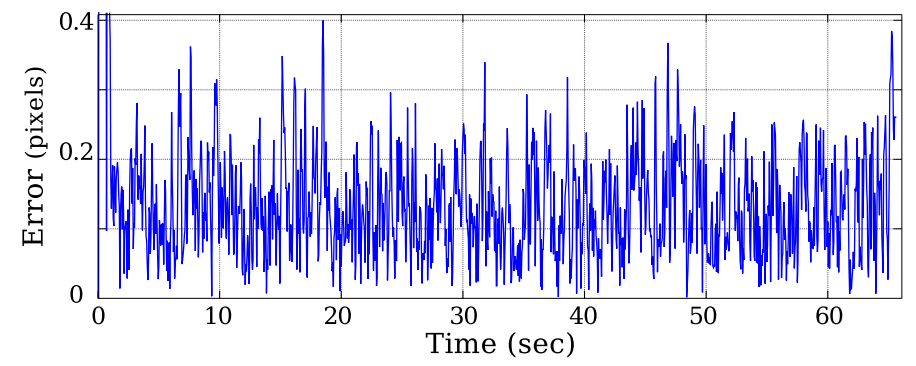

(b) With white noise.

Figure 12. 2D trajectories tracking error $(N=3):\|q-w\|$. 


\section{CONCLUSION}

The proposed MRI-based ferromagnetic microcapsule steering and navigation strategy has been developed at milliscale and microscale, where low-level multiplexed controllers and observers have been designed. The main drawback of MRI-based navigation stems from the strong limitations on the magnetic gradient amplitude of available MRI devices. As magnetic forces used for propelling are volumetric, whereas the drag force is at best dependent on the microcapsules area, the smaller the capsule, the higher the required control forces with respect to hydrodynamic perturbations. Consequently, this approach is well conditioned for microcapsules whose radius is up to a few dozen micrometers with actual MRI devices. Targeting aims at focusing these micro-carriers and stopping them through embolization at the arterioles entry close to the occluded blood vessels. Possible releasing mechanisms could rely on biodegradable polymer and techniques used in hyperthermia where aggregates of nanocapsules can be heated to melt polymer. Such a solution is actually under experimentation for validation of the proposed minimally invasive MRI-based microrobotic system.

\section{ACKNOWLEDGMENTS}

This work was supported by European Union's 7th Framework Program and its research area ICT-2007.3.6 Micro/nanosystems under the project ICT-224594 entitled NANOMA (Nano-Actuactors and Nano-Sensors for Medical Applications). 


\section{References}

[1] M. S. K. Ishiyama and K. I. Arai, "Magnetic micromachines for medical applications," Journal of Magnetism and Magnetic Materials, vol. 242-245, pp. 41-46, 2002, doi = "10.1016/j.jmmm.2006.10.1195", issn = "0304-8853", publisher=Elsevier.

[2] J. Abbott, Z. Nagy, F. Beyeler, and B. Nelson, "Robotics in the small," p. 92, 2007.

[3] T. A. Cavalcanti, B. Shirinzadeh and S. Ikeda, "Nanorobot for Brain Aneurysm," International Journal of Robotic Research, vol. 28, no. 4, pp. 558-570, 2009.

[4] J. J. Abbott, K. E. Peyer, M. C. Lagomarsino, L. Zhang, L. X. Dong, I. K. Kaliakatsos, and B. J. Nelson, "How should microrobots swim?" International Journal of Robotics Research, July 2009, doi:10.1177/0278364909341658.

[5] B. Behkam and M. Sitti, "Design methodology for biomimetic propulsion of miniature swimming robots," ASME J. Dyn. Syst. Meas. Control, p. 3643, 2006.

[6] M. M. O. F. Z. L. Martel, S. and P. Pouponneau, "Flagellated magnetotactic bacteria as controlled MRItrackable propulsion and steering systems for medical nanorobots operating in the human microvasculature," Int. J. Robot. Res., vol. 28, no. 4, p. 571582 , 2009.

[7] O. Felfoul, J. Mathieu, G. Beaudoin, and S. Martel, "In vivo MR-tracking based on magnetic signature selective excitation,” vol. 27, no. 1, pp. 28-35, Jan. 2008.

[8] K. M. Sabra W. and M. S., "Use of 3D potential field and an enhanced breath-first search algorithms for path-planning of microdevices propelled in the cardiovascular system," 27th IEEE EMBS Annual International Conference, pp. 91 - 100, 2005.

[9] P. S. and P. J-O, "Frontier research program on biomedical robot for intravascular therapy," 2nd IEEE/RAS-EMBS International Conference on Biomedical Robotics and Biomechatronics, pp. $360-365,2008$. 
[10] S. Tamaz, A. Chanu, J.-B. Mathieu, R. Gourdeau, and S. Martel, "Real-time mri-based control of a ferromagnetic core for endovascular navigation," vol. 55, no. 7, pp. 18541863, July 2008.

[11] C. Kirbas and F. Quek, "A review of vessel extraction techniques and algorithms," ACM Comput. Surv., vol. 36, no. 2, pp. 81-121, 2004.

[12] P. Reuzé, J. Coatrieux, L. Luo, and J. Dillenseger, "A 3D moment based approach for blood vessel detection and quantification in MRA," Technology and Health Care, vol. 1, pp. 181-188, 1993.

[13] J. Tokuda, G. S. Fischer, S. P. DiMaio, D. G. Gobbi, C. Csoma, P. W. Mewes, G. Fichtinger, C. M. Tempany, and N. Hata, "Integrated navigation and control software system for mri-guided robotic prostate interventions," Computerized Medical Imaging and Graphics, vol. 34, no. 1, pp. 3-8, 2010, image-Guided Surgical Planning and Therapy.

[14] T. Deschamps, "Curve and shape extraction with minimal path and level-sets techniques: Applications to 3d medical imaging," Ph.D. dissertation, University of Paris Dauphine, 2001.

[15] O. Wink, W. J. Niessen, and M. A. Viergever, "Minimum cost path determination using a simple heuristic function," Int. Conf. on Pattern Recognition, vol. 3, p. 7010, 2000.

[16] O. Wink, W. Niessen, B. Verdonck, and M. Viergever, "Vessel axis determination using wave front propagation analysis," Medical Image Computing and Computer-Assisted Intervention, vol. 2208, pp. 845-853, 2001, lecture Notes in Computer Science.

[17] L. Cohen and R. Kimmel, "Global minimum for active contour models: A minimal path approach," Int. J. of Computer Vision, vol. 24, no. 1, pp. 57-78, 1997.

[18] E. W. Dijkstra, "A note on two problems in connexion with graphs," Numerische Mathematik, vol. 1, p. 269?271, 1959. 
[19] J. Pearl, Heuristics: intelligent search strategies for computer problem solving. Boston, MA, USA: Addison-Wesley Longman Publishing Co., Inc., 1984.

[20] J. Sethian et al., Level set methods and fast marching methods. Cambridge university press Cambridge, 1999.

[21] A. Frangi, W. Niessen, K. Vincken, and M. Viergever, "Multiscale vessel enhancement filtering," Lecture Notes in Computer Science, pp. 130-137, 1998.

[22] L. Arcèse, M. Fruchard, and A. Ferreira, "Nonlinear modeling and robust controllerobserver for a magnetic microrobot in a fluidic environment using MRI gradients," in IEEE/RSJ Int. Conf. on Intel. Robots and Systems, St. Louis, MO, USA, Oct. 2009, pp. 534-539.

[23] D. Clarke, C. Mohtadi, and P. Tuffs, "Generalized predictive control — Part I. The Basic algorithm," Automatica, vol. 23, pp. 137-160, 1987.

[24] —_, "Generalized predictive control — part II. Extensions and interpretations." Automatica, vol. 23, no. 2, pp. 149-160, 1987.

[25] M. Mahfouf and D. Linkens, Generalised predictive control and bioengineering, ser. Systems and Control. CRC press, August 1998.

[26] S. Huang, K. Tan, and T. Lee, Applied predictive control, ser. Advances in Industrial Control. Springer Verlag, 2002.

[27] S. Qin and T. Badgwell, "A survey of industrial model predictive control technology," Control engineering practice, vol. 11, no. 7, pp. 733-764, 2003.

[28] P. Boucher and D. Dumur, La commande prédictive, TECHNIP ed., ser. Collection Mthodes et Pratiques de 1?Ingnieur, Paris, 1996. 